\title{
Solvability of a class of product-type systems of difference equations
}

\section{Stevo Stević}

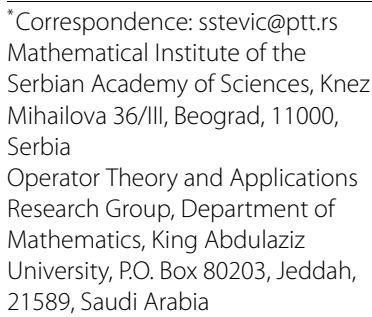

\begin{abstract}
A solvable class of product-type systems of difference equations with two dependent variables on the complex domain is presented. The main results complement some recent ones in the literature, while their proofs contain some refined methodological details. We provide closed form formulas for general solutions to the system or give procedures for how to get them.
\end{abstract}

MSC: 39A20; 39A45

Keywords: system of difference equations; product-type system; solvable system; solutions in closed form

\section{Introduction}

Nonlinear difference equations and systems have been studied a lot in the last few decades (see, e.g., [1-29]). Two of the topics of recent interest are symmetric and closely related systems (see, for example, [3, 6-12, 15, 16, 19, 20, 22, 23, 26-29]), whose investigation was considerably influenced by some papers by Papaschinopoulos and Schinas (see, for example, [8-10]), and the solvable difference equations and systems (see, for example, [3, 14, 18-22, 24-29] and the references therein). For some classical methods for solving the equations and systems see, for example, [1,30-33]. It has been shown recently that many nonlinear equations and systems can be solved by transforming them to linear ones (see, for example, $[3,14,18,21,24,25]$ and the related references therein).

Some of the equations and systems that we have studied recently, such as the ones in [17] and [23] (see also [13]), are obtained by adding constants to the right-hand sides of some product-type equations/systems or by taking the maximum of some constants and the right-hand sides of the equations/systems. This means that they are related to the product-type ones, which are usually some kind of boundary cases. The case of positive initial values and multipliers is simple, since in that case the equations/systems can easily be treated by one of the simplest transformation methods. The case of complex initial values is more complex due to the fact that complex functions need not be single valued. Hence, our methods in $[3,18,21]$ and other related papers cannot be applied. This has motivated us to start investigating product-type systems on the complex domain. In a series of papers, see [19, 20, 22, 26-29], we have obtained some results in the area (during the study of the equation in [21] we came across a product-type equation). In our first papers on the topic (see $[20,22,26])$ the systems have not had coefficients different from

(c) The Author(s) 2016. This article is distributed under the terms of the Creative Commons Attribution 4.0 International License (http://creativecommons.org/licenses/by/4.0/), which permits unrestricted use, distribution, and reproduction in any medium, provided you give appropriate credit to the original author(s) and the source, provide a link to the Creative Commons license, and indicate if changes were made. 
one. However, not long after that we have introduced two coefficients and also got solvable systems (this was done for the first time in [19], and somewhat later in [27-29]). We have also observed the fact that there are only a few solvable product-type systems of difference equations with two dependent variables. Hence, our aim is to describe all such producttype systems and present formulas for the general solutions for each of them.

This paper complements our previous results on the solvability of product-type systems of difference equations with two dependent variables, by studying the following one:

$$
z_{n}=\alpha z_{n-1}^{a} w_{n-2}^{b}, \quad w_{n}=\beta w_{n-2}^{c} z_{n-1}^{d}, \quad n \in \mathbb{N}_{0},
$$

where $a, b, c, d \in \mathbb{Z}, \alpha, \beta \in \mathbb{C}$ and $z_{-1}, w_{-2}, w_{-1} \in \mathbb{C}$. To do this we will modify ideas and methods from our previous papers, for example, the ones in [19, 27-29].

If the initial values belong to the following set:

$$
\left\{\left(z_{-1}, w_{-2}, w_{-1}\right) \in \mathbb{C}^{3}: z_{-1}=0 \text { or } w_{-2}=0 \text { or } w_{-1}=0\right\}
$$

and if some of the exponents $a, b, c, d$ are negative, then such solutions are not defined. Hence, this set of initial values will not be taken into consideration. Besides, if $\alpha=0$ or $\beta=0$, we get $z_{n}=0$ and $w_{n}=0$ for every $n \in \mathbb{N}_{0}$, respectively, which are quite simple cases, or also obtain solutions which are not well defined, so, we will also assume that $\alpha \neq 0 \neq \beta$. We use the convention $\sum_{i=k}^{l} a_{i}=0$, when $l<k$, throughout the paper.

\section{Main results}

Our main results are presented here. The following three cases will be considered separately:

(i) $c=0, a c=b d$;

(ii) $c \neq 0, a c=b d$;

(iii) $a c \neq b d$.

Clearly, in case (i) from $c=0$ and $a c=b d$ it immediately follows that $b d=0$, but we have chosen to write $a c=b d$ at this point, to point out that the whole analysis essentially depends on the values of the quantities $c$ and $a c-b d$, that is, if they are equal to zero or not.

First, we will consider case (i), then case (iii) and at the end case (ii), for the presentational reasons.

Theorem 1 Assume that $a, b, d \in \mathbb{Z}, c=0, b d=0, \alpha, \beta \in \mathbb{C} \backslash\{0\}$ and $z_{-1}, w_{-2}, w_{-1} \in \mathbb{C} \backslash\{0\}$. Then the following statements hold.

(a) If $a \neq 1$, then the general solution to system (1) is given by the following formulas:

$$
z_{n}=\alpha^{\frac{1-a^{n+1}}{1-a}} \beta^{b \frac{1-a^{n-1}}{1-a}} z_{-1}^{a^{n+1}} w_{-2}^{b a^{n}} w_{-1}^{b a^{n-1}}, \quad n \geq 2,
$$

and

$$
w_{n}=\alpha^{d \frac{1-a^{n}}{1-a}} \beta z_{-1}^{d a^{n}}, \quad n \geq 2 .
$$


(b) If $a=1$, then the general solution to system (1) is given by the following formulas:

$$
z_{n}=\alpha^{n+1} \beta^{b(n-1)} z_{-1} w_{-2}^{b} w_{-1}^{b}, \quad n \geq 2
$$

and

$$
w_{n}=\alpha^{d n} \beta z_{-1}^{d}, \quad n \geq 2
$$

Proof Since $c=0$, we have

$$
z_{n}=\alpha z_{n-1}^{a} w_{n-2}^{b}, \quad w_{n}=\beta z_{n-1}^{d}, \quad n \in \mathbb{N}_{0} .
$$

From (6) and $b d=0$ is obtained

$$
z_{n}=\alpha \beta^{b} z_{n-1}^{a}, \quad n \geq 2 .
$$

From (7) we easily get

$$
z_{n}=\left(\alpha \beta^{b}\right)^{\sum_{j=0}^{n-2} a^{j}} z_{1}^{a^{n-1}}, \quad n \geq 2,
$$

which, along with

$$
z_{1}=\alpha^{1+a} z_{-1}^{a^{2}} w_{-2}^{a b} w_{-1}^{b}
$$

yields

$$
z_{n}=\alpha^{\sum_{j=0}^{n} a^{j}} \beta^{b \sum_{j=0}^{n-2} a^{j}} z_{-1}^{a^{n+1}} w_{-2}^{b a^{n}} w_{-1}^{b a^{n-1}}, \quad n \geq 2 .
$$

By using equation (9) along with the formula for the sum of the geometric progression we see that equation (2) holds when $a \neq 1$, while equation (4) is directly obtained for $a=1$. From (9), the equality $w_{n}=\beta z_{n-1}^{d}$, and the condition $b d=0$, we obtain

$$
\begin{aligned}
w_{n} & =\alpha^{d \sum_{j=0}^{n-1} a^{j}} \beta^{1+b d \sum_{j=0}^{n-3} a^{j}} z_{-1}^{d a^{n}} w_{-2}^{b d a^{n-1}} w_{-1}^{b d a^{n-2}} \\
& =\alpha^{d \sum_{j=0}^{n-1} a^{j}} \beta z_{-1}^{d a^{n}}, \quad n \geq 2 .
\end{aligned}
$$

By using equation (10) along with the formula for the sum of the geometric progression we see that equation (3) holds when $a \neq 1$, while equation (5) is directly obtained for $a=1$, completing the proof of the theorem.

Theorem 2 Assume that $a, b, c, d \in \mathbb{Z}, a c \neq b d, \alpha, \beta \in \mathbb{C} \backslash\{0\}$ and $z_{-1}, w_{-2}, w_{-1} \in \mathbb{C} \backslash\{0\}$. Then system (1) is solvable in closed form.

Proof Since $\alpha, \beta \in \mathbb{C} \backslash\{0\}$ and $z_{-1}, w_{-2}, w_{-1} \in \mathbb{C} \backslash\{0\}$, using (1) it easily follows that $z_{n} \neq 0$ for $n \geq-1$ and $w_{n} \neq 0$ for $n \geq-2$. Thus, from (1) we have

$$
w_{n-2}^{b}=\frac{z_{n}}{\alpha z_{n-1}^{a}}, \quad n \in \mathbb{N}_{0},
$$


and

$$
w_{n}^{b}=\beta^{b} w_{n-2}^{b c} z_{n-1}^{b d}, \quad n \in \mathbb{N}_{0}
$$

From (11) and (12) we get

$$
z_{n+2}=\alpha^{1-c} \beta^{b} z_{n+1}^{a} z_{n}^{c} z_{n-1}^{b d-a c}, \quad n \in \mathbb{N}_{0} .
$$

Let $\mu=\alpha^{1-c} \beta^{b}$,

$$
a_{1}=a, \quad b_{1}=c, \quad c_{1}=b d-a c, \quad y_{1}=1 .
$$

Then

$$
z_{n+2}=\mu^{y_{1}} z_{n+1}^{a_{1}} z_{n}^{b_{1}} z_{n-1}^{c_{1}}, \quad n \in \mathbb{N}_{0} .
$$

From (15) is obtained

$$
\begin{aligned}
z_{n+2} & =\mu^{y_{1}}\left(\mu z_{n}^{a_{1}} z_{n-1}^{b_{1}} z_{n-2}^{c_{1}}\right)^{a_{1}} z_{n}^{b_{1}} z_{n-1}^{c_{1}} \\
& =\mu^{y_{1}+a_{1}} z_{n}^{a_{1} a_{1}+b_{1}} z_{n-1}^{b_{1} a_{1}+c_{1}} z_{n-2}^{c_{1} a_{1}} \\
& =\mu^{y_{2}} z_{n}^{a_{2}} z_{n-1}^{b_{2}} z_{n-2}^{c_{2}},
\end{aligned}
$$

for $n \in \mathbb{N}$, where

$$
a_{2}:=a_{1} a_{1}+b_{1}, \quad b_{2}:=b_{1} a_{1}+c_{1}, \quad c_{2}:=c_{1} a_{1}, \quad y_{2}:=y_{1}+a_{1} \text {. }
$$

Suppose that

$$
z_{n+2}=\mu^{y_{k}} z_{n+2-k}^{a_{k}} z_{n+1-k}^{b_{k}} z_{n-k}^{c_{k}}
$$

for a $k \in \mathbb{N}$ and every $n \geq k-1$, and

$$
\begin{aligned}
& a_{k}=a_{1} a_{k-1}+b_{k-1}, \quad b_{k}=b_{1} a_{k-1}+c_{k-1}, \quad c_{k}=c_{1} a_{k-1}, \\
& y_{k}:=y_{k-1}+a_{k-1} .
\end{aligned}
$$

From (15) with $n \rightarrow n-k$ and (18), it follows that

$$
\begin{aligned}
z_{n+2} & =\mu^{y_{k}}\left(\mu z_{n+1-k}^{a_{1}} z_{n-k}^{b_{1}} z_{n-k-1}^{c_{1}}\right)^{a_{k}} z_{n+1-k}^{b_{k}} z_{n-k}^{c_{k}} \\
& =\mu^{y_{k}+a_{k}} z_{n+1-k}^{a_{1} a_{k}+b_{k}} z_{n-k}^{b_{1} a_{k}+c_{k}} z_{n-k-1}^{c_{1} a_{k}} \\
& =\mu^{y_{k+1}} z_{n+1-k}^{a_{k+1}} z_{n-k}^{b_{k+1}} z_{n-k-1}^{c_{k+1}},
\end{aligned}
$$

for $n \geq k$, where

$$
\begin{aligned}
& a_{k+1}:=a_{1} a_{k}+b_{k}, \quad b_{k+1}:=b_{1} a_{k}+c_{k}, \quad c_{k+1}:=c_{1} a_{k}, \\
& y_{k+1}:=y_{k}+a_{k} .
\end{aligned}
$$


Equalities (16), (17), (21), (22), (23), and the induction show that (18), (19), and (20), hold for every $k, n \in \mathbb{N}$ such that $2 \leq k \leq n+1$. Note that (18) holds also for $k=1$.

For $k=n+1,(18)$ becomes

$$
\begin{aligned}
z_{n+2}= & \mu^{y_{n+1}} z_{1}^{a_{n+1}} z_{0}^{b_{n+1}} z_{-1}^{c_{n+1}} \\
= & \left(\alpha^{1-c} \beta^{b}\right)^{y_{n+1}}\left(\alpha^{1+a} z_{-1}^{a^{2}} w_{-2}^{a b} w_{-1}^{b}\right)^{a_{n+1}}\left(\alpha z_{-1}^{a} w_{-2}^{b}\right)^{b_{n+1}} z_{-1}^{c_{n+1}} \\
= & \alpha^{(1-c) y_{n+1}+(1+a) a_{n+1}+b_{n+1}} \beta^{b y_{n+1}} z_{-1}^{a^{2} a_{n+1}+a b_{n+1}+c_{n+1}} \\
& \times w_{-2}^{a b a_{n+1}+b b_{n+1}} w_{-1}^{b a_{n+1}} \\
= & \alpha^{y_{n+3}-c y_{n+1}} \beta^{b y_{n+1}} z_{-1}^{a_{n+3}-c a_{n+1}} w_{-2}^{b a_{n+2}} w_{-1}^{b a_{n+1}}, \quad n \in \mathbb{N}_{0},
\end{aligned}
$$

where we have also used the fact $z_{0}=\alpha z_{-1}^{a} w_{-2}^{b}$, (8), (19), and (20).

Further note that (19) implies that $\left(a_{k}\right)_{k \geq 4}$ is a solution to the equation

$$
a_{k}=a_{1} a_{k-1}+b_{1} a_{k-2}+c_{1} a_{k-3}
$$

Since $c_{1} \neq 0$, from $(25)$, we get

$$
a_{k-3}=\frac{a_{k}-a_{1} a_{k-1}-b_{1} a_{k-2}}{c_{1}},
$$

from which it follows that we can calculate $a_{l}$ for every non-positive $l$, that is, for $k \leq 3$. A direct calculation shows that

$$
a_{0}=1, \quad a_{-1}=a_{-2}=0
$$

Moreover, it is shown that $\left(a_{k}\right)_{k \geq-2},\left(b_{k}\right)_{k \geq-2},\left(c_{k}\right)_{k \geq-2}$ are solutions to (25) such that

$$
\begin{array}{lll}
a_{-2}=0, & a_{-1}=0, & a_{0}=1 ; \\
b_{-2}=0, & b_{-1}=1, & b_{0}=0 ; \\
c_{-2}=1, & c_{-1}=0, & c_{0}=0 ;
\end{array}
$$

respectively, whereas $\left(y_{k}\right)_{k \geq-2}$ satisfies (20) and

$$
y_{-2}=y_{-1}=y_{0}=0, \quad y_{1}=1
$$

From (20) and since $a_{0}=1$, we get

$$
y_{k}=\sum_{j=0}^{k-1} a_{j} .
$$

The solvability of (25) shows that for $\left(a_{k}\right)_{k \geq-2}$ can be found a closed form formula. Therefore, using equation (29) and known formulas for the following sums:

$$
s_{m}^{(j)}(\zeta)=\sum_{k=1}^{m} k^{j} \zeta^{k}, \quad m \in \mathbb{N}_{0}
$$


where $j=\overline{0,2}$ (see, e.g., $[31,33]$ ), the closed form formula for $\left(y_{k}\right)_{k \in \mathbb{N}}$ is easily obtained. This along with (24) shows the solvability of (13).

From (1), we also have

$$
z_{n-1}^{d}=\frac{w_{n}}{\beta w_{n-2}^{c}}, \quad n \in \mathbb{N}_{0},
$$

and

$$
z_{n}^{d}=\alpha^{d} z_{n-1}^{a d} w_{n-2}^{b d}, \quad n \in \mathbb{N}_{0} .
$$

Equalities (31) and (32) yield

$$
w_{n+1}=\alpha^{d} \beta^{1-a} w_{n}^{a} w_{n-1}^{c} w_{n-2}^{b d-a c}, \quad n \in \mathbb{N}_{0} .
$$

We have

$$
w_{0}=\beta w_{-2}^{c} z_{-1}^{d} \text {. }
$$

Similarly as above we get

$$
w_{n+1}=\eta^{y_{k}} w_{n+1-k}^{a_{k}} w_{n-k}^{b_{k}} w_{n-k-1}^{c_{k}}, \quad n \geq k-1,
$$

where $\eta=\alpha^{d} \beta^{1-a},\left(a_{k}\right)_{k \in \mathbb{N}},\left(b_{k}\right)_{k \in \mathbb{N}}$ and $\left(c_{k}\right)_{k \in \mathbb{N}}$ are defined by (14) and (19), whereas $\left(y_{k}\right)_{k \in \mathbb{N}}$ satisfies (20) and (28), so it is given by (29).

From (35) with $k=n+1$ and (34) is obtained

$$
\begin{aligned}
w_{n+1} & =\eta^{y_{n+1}} w_{0}^{a_{n+1}} w_{-1}^{b_{n+1}} w_{-2}^{c_{n+1}} \\
& =\left(\alpha^{d} \beta^{1-a}\right)^{y_{n+1}}\left(\beta w_{-2}^{c} z_{-1}^{d}\right)^{a_{n+1}} w_{-1}^{b_{n+1}} w_{-2}^{c_{n+1}} \\
& =\alpha^{d y_{n+1}} \beta^{(1-a) y_{n+1}+a_{n+1}} z_{-1}^{d a_{n+1}} w_{-2}^{c a_{n+1}+c_{n+1}} w_{-1}^{b_{n+1}} \\
& =\alpha^{d y_{n+1}} \beta^{y_{n+2}-a y_{n+1}} z_{-1}^{d a_{n+1}} w_{-2}^{a_{n+3}-a a_{n+2}} w_{-1}^{a_{n+2}-a a_{n+1}},
\end{aligned}
$$

for $n \in \mathbb{N}_{0}$.

The solvability of (25) along with (27) shows that for $\left(a_{k}\right)_{k \geq-2}$ we can find a closed form formula, from which along with (29) the formulas for $y_{k}$ can also be obtained, as described above. These facts along with (36) imply the solvability of equation (33). It is not difficult to show that formulas (24) and (36) really represent solutions to system (1). Thus, system (1) is also solvable in this case, as claimed.

Corollary 1 Consider system (1) with $a, b, c, d \in \mathbb{Z}, a c \neq b d, \alpha, \beta \in \mathbb{C} \backslash\{0\}$, and $z_{-1}, w_{-2}, w_{-1} \in \mathbb{C} \backslash\{0\}$. Then the general solution to system (1) is given by (24) and (36), where $\left(a_{k}\right)_{k \in \mathbb{N}}$ satisfies equation (25) with initial conditions (27), while $\left(y_{k}\right)_{k \in \mathbb{N}}$ is given by (29) and can be found by using formulas for the sums in (30).

Theorem 3 Assume that $a, b, c, d \in \mathbb{Z}, c \neq 0, a c=b d, \alpha, \beta \in \mathbb{C} \backslash\{0\}$ and $z_{-1}, w_{-2}, w_{-1} \in$ $\mathbb{C} \backslash\{0\}$. Then the following statements hold. 
(a) If $a^{2} \neq-4 c$ and $a+c \neq 1$, then the general solution to system (1) is given by the following formulas:

$$
\begin{aligned}
& z_{n}=\alpha^{\frac{a\left(\lambda_{2}-1\right) \lambda_{1}^{n+1}-a\left(\lambda_{1}-1\right) \lambda_{2}^{n+1}+\left(\lambda_{1}-\lambda_{2}\right)(1-c)}{\left(\lambda_{1}-1\right)\left(\lambda_{2}-1\right)\left(\lambda_{1}-\lambda_{2}\right)}} \beta^{b \frac{\left(\lambda_{2}-1\right) \lambda_{1}^{n}-\left(\lambda_{1}-1\right) \lambda_{2}^{n}+\lambda_{1}-\lambda_{2}}{\left(\lambda_{1}-1\right)\left(\lambda_{2}-1\right)\left(\lambda_{1}-\lambda_{2}\right)}} \\
& \times z_{-1}^{a \frac{\lambda_{1}^{n+1}-\lambda_{2}^{n+1}}{\lambda_{1}-\lambda_{2}}} w_{-2}^{b \frac{\lambda_{1}^{n+1}-\lambda_{2}^{n+1}}{\lambda_{1}-\lambda_{2}}} w_{-1}^{b \frac{\lambda_{1}^{n}-\lambda_{2}^{n}}{\lambda_{1}-\lambda_{2}}}
\end{aligned}
$$

and

$$
\begin{aligned}
& w_{n}=\alpha^{d \frac{\left(\lambda_{2}-1\right) \lambda_{1}^{n+1}-\left(\lambda_{1}-1\right) \lambda_{2}^{n+1}+\lambda_{1}-\lambda_{2}}{\left(\lambda_{1}-1\right)\left(\lambda_{2}-1\right)\left(\lambda_{1}-\lambda_{2}\right)}} \beta^{\frac{c\left(\lambda_{2}-1\right) \lambda_{1}^{n}-c\left(\lambda_{1}-1\right) \lambda_{2}^{n}+\left(\lambda_{1}-\lambda_{2}\right)(1-a)}{\left(\lambda_{1}-1\right)\left(\lambda_{2}-1\right)\left(\lambda_{1}-\lambda_{2}\right)}} \\
& \times z_{-1}^{d \frac{\lambda_{1}^{n+1}-\lambda_{2}^{n+1}}{\lambda_{1}-\lambda_{2}}} w_{-2} w^{\frac{\lambda_{1}^{n+1}-\lambda_{2}^{n+1}}{\lambda_{1}-\lambda_{2}}} w_{-1}^{c{\frac{\lambda}{\lambda_{1}-\lambda_{2}}}^{n}},
\end{aligned}
$$

where

$$
\lambda_{1,2}=\frac{a+\sqrt{a^{2}+4 c}}{2} .
$$

(b) If $a^{2} \neq-4 c$ and $a+c=1$, then the general solution to system (1) is given by the following formulas:

$$
z_{n}=\alpha^{\frac{a \lambda_{1}^{n+1}+((c-1) n-2) \lambda_{1}+(1-c) n+1+c}{\left(1-\lambda_{1}\right)^{2}}} \beta^{b \frac{n-1-n \lambda_{1}+\lambda_{1}^{n}}{\left(1-\lambda_{1}\right)^{2}}} z_{-1}^{a \frac{\lambda_{1}^{n+1}-1}{\lambda_{1}-1}} w_{-2}^{b \frac{\lambda_{1}^{n+1}-1}{\lambda_{1}-1}} w_{-1}^{b \frac{\lambda_{1}^{n}-1}{\lambda_{1}-1}}
$$

and

$$
\begin{aligned}
w_{n}= & \alpha \frac{d \frac{n-(n+1) \lambda_{1}+\lambda_{1}^{n+1}}{\left(1-\lambda_{1}\right)^{2}}}{\beta} \beta^{\frac{c \lambda_{1}^{n}+((a-1) n+a-2) \lambda_{1}+(1-a) n+1}{\left(1-\lambda_{1}\right)^{2}}} \\
& \times z_{-1}^{d \frac{\lambda_{1}^{n+1}-1}{\lambda_{1}-1}} w_{-2}^{c}{ }^{c \lambda_{1}^{n+1}-1} w_{1^{-1}}^{c} w_{-1}^{\lambda_{1}^{n}-1}
\end{aligned}
$$

where

$$
\lambda_{1}=-c \text {. }
$$

(c) If $a^{2}=-4 c$ and $a+c \neq 1$, then the general solution to system (1) is given by the following formulas:

$$
\begin{aligned}
z_{n}= & \alpha \frac{a n \lambda_{1}^{n+1}-a(n+1) \lambda_{1}^{n}+1-c}{\left(1-\lambda_{1}\right)^{2}} \beta^{b \frac{1-n \lambda_{1}^{n-1}+(n-1) \lambda_{1}^{n}}{\left(1-\lambda_{1}\right)^{2}}} \\
& \times z_{-1}^{a(n+1) \lambda_{1}^{n}} w_{-2}^{b(n+1) \lambda_{1}^{n}} w_{-1}^{b n \lambda_{1}^{n-1}}
\end{aligned}
$$

and

$$
\begin{aligned}
w_{n}= & \alpha^{d \frac{1-(n+1) \lambda_{1}^{n}+n \lambda_{1}^{n+1}}{\left(1-\lambda_{1}\right)^{2}}} \beta^{\frac{c(n-1) \lambda_{1}^{n}-c n \lambda_{1}^{n-1}+1-a}{\left(1-\lambda_{1}\right)^{2}}} \\
& \times z_{-1}^{d(n+1) \lambda_{1}^{n}} w_{-2}^{c(n+1) \lambda_{1}^{n}} w_{-1}^{c n \lambda_{1}^{n-1}}
\end{aligned}
$$


where

$$
\lambda_{1}=\frac{a}{2}
$$

(d) If $a^{2}=-4 c$ and $a+c=1$, then the general solution to system (1) is given by the following formulas:

$$
z_{n}=\alpha^{\frac{(1-c) n^{2}+(c+3) n+2}{2}} \beta^{b \frac{(n-1) n}{2}} z_{-1}^{a(n+1)} w_{-2}^{b(n+1)} w_{-1}^{b n}
$$

and

$$
w_{n}=\alpha^{d \frac{n(n+1)}{2}} \beta^{\frac{(n+1)((1-a) n+2)}{2}} z_{-1}^{d(n+1)} w_{-2}^{c(n+1)} w_{-1}^{c n} .
$$

Proof Similar to the proof of Theorem 2 we have $z_{n} \neq 0$ for $n \geq-1$ and $w_{n} \neq 0$ for $n \geq-2$, and for every such solution (13) holds, from which, along with the condition $a c=b d$, it follows that

$$
z_{n+2}=\alpha^{1-c} \beta^{b} z_{n+1}^{a} z_{n}^{c}
$$

for $n \in \mathbb{N}_{0}$.

$$
\text { Let } v=\alpha^{1-c} \beta^{b} \text {, }
$$

$$
\hat{a}_{1}=a, \quad \hat{b}_{1}=c, \quad \hat{y}_{1}=1 .
$$

Then

$$
z_{n+2}=v^{\hat{y}_{1}} z_{n+1}^{\hat{a}_{1}} z_{n}^{\hat{b}_{1}}
$$

for $n \in \mathbb{N}_{0}$.

From (50) is obtained

$$
\begin{aligned}
z_{n+2} & =v^{\hat{y}_{1}}\left(v z_{n}^{\hat{a}_{1}} z_{n-1}^{\hat{b}_{1}}\right)^{\hat{a}_{1}} z_{n}^{\hat{b}_{1}} \\
& =v^{\hat{y}_{1}} \hat{a}_{1} z_{n}^{\hat{a}_{1} \hat{a}_{1}+\hat{b}_{1}} z_{n-1}^{\hat{b}_{1} \hat{a}_{1}} \\
& =v^{\hat{y}_{2}} z_{n}^{\hat{a}_{2}} z_{n-1}^{\hat{b}_{2}},
\end{aligned}
$$

for $n \in \mathbb{N}$, where

$$
\hat{a}_{2}:=\hat{a}_{1} \hat{a}_{1}+\hat{b}_{1}, \quad \hat{b}_{2}:=\hat{b}_{1} \hat{a}_{1}, \quad \hat{y}_{2}:=\hat{y}_{1}+\hat{a}_{1} .
$$

Suppose that

$$
z_{n+2}=v^{\hat{y}_{k}} z_{n+2-k}^{\hat{a}_{k}} z_{n+1-k}^{\hat{k}_{k}}
$$

holds for some $k \in \mathbb{N}$ and for every $n \geq k-1$, and that

$$
\hat{a}_{k}=\hat{a}_{1} \hat{a}_{k-1}+\hat{b}_{k-1}, \quad \hat{b}_{k}=\hat{b}_{1} \hat{a}_{k-1}, \quad \hat{y}_{k}:=\hat{y}_{k-1}+\hat{a}_{k-1} .
$$


Then from (50), with $n \rightarrow n-k$ and (53), we get

$$
\begin{aligned}
z_{n+2} & =v^{\hat{y}_{k}}\left(v z_{n+1-k}^{\hat{a}_{1}} z_{n-k}^{\hat{b}_{1}}\right)^{\hat{a}_{k}} z_{n+1-k}^{\hat{b}_{k}} \\
& =v^{\hat{y}_{k}+\hat{a}_{k}} z_{n+1-k}^{\hat{a}_{1} \hat{a}_{k}+\hat{b}_{k}} z_{n-k}^{\hat{b}_{1} \hat{a}_{k}} \\
& =v^{\hat{y}_{k+1}} z_{n+1-k}^{\hat{a}_{k+1}} z_{n-k}^{\hat{b}_{k+1}},
\end{aligned}
$$

for $n \geq k$, where

$$
\hat{a}_{k+1}:=\hat{a}_{1} \hat{a}_{k}+\hat{b}_{k}, \quad \hat{b}_{k+1}:=\hat{b}_{1} \hat{a}_{k}, \quad \hat{y}_{k+1}:=\hat{y}_{k}+\hat{a}_{k}
$$

Equalities (51), (52), (55), (56), and the induction show that (53) and (54), hold for every $k, n \in \mathbb{N}$ such that $2 \leq k \leq n+1$. Note that the equality in (53) also holds for $k=1$ (see (50)).

For $k=n+1$, (53) becomes

$$
\begin{aligned}
z_{n+2}= & v^{\hat{y}_{n+1}} z_{1}^{\hat{a}_{n+1}} z_{0}^{\hat{b}_{n+1}} \\
= & \left(\alpha^{1-c} \beta^{b}\right)^{\hat{y}_{n+1}}\left(\alpha^{1+a} z_{-1}^{a^{2}} w_{-2}^{a b} w_{-1}^{b}\right)^{\hat{a}_{n+1}}\left(\alpha z_{-1}^{a} w_{-2}^{b}\right)^{\hat{b}_{n+1}} \\
= & \alpha^{(1-c) \hat{y}_{n+1}+(1+a) \hat{a}_{n+1}+\hat{b}_{n+1}} \beta^{b \hat{y}_{n+1}} z_{-1}^{a^{2} \hat{a}_{n+1}+a \hat{b}_{n+1}} \\
& \times w_{-2}^{a b \hat{a}_{n+1}+b \hat{b}_{n+1}} w_{-1}^{b \hat{a}_{n+1}} \\
= & \alpha^{\hat{y}_{n+3}-c \hat{y}_{n+1}} \beta^{b \hat{y}_{n+1}} z_{-1}^{a \hat{a}_{n+2}} w_{-2}^{b \hat{a}_{n+2}} w_{-1}^{b \hat{a}_{n+1}}, \quad n \in \mathbb{N}_{0},
\end{aligned}
$$

where we have used the fact $z_{0}=\alpha z_{-1}^{a} w_{-2}^{b},(8)$, and (54).

Now note that $\left(\hat{a}_{k}\right)_{k \geq 3}$ is a solution to the equation

$$
\hat{a}_{k}=\hat{a}_{1} \hat{a}_{k-1}+\hat{b}_{1} \hat{a}_{k-2} \text {. }
$$

As in the proof of Theorem 2, it is shown that $\left(\hat{a}_{k}\right)_{k \geq-2}$ and $\left(\hat{b}_{k}\right)_{k \geq-2}$ are solutions to equation (58), satisfying the initial conditions

$$
\begin{array}{ll}
\hat{a}_{-1}=0, & \hat{a}_{0}=1 ; \\
\hat{b}_{-1}=1, & \hat{b}_{0}=0,
\end{array}
$$

respectively, whereas $\left(\hat{y}_{k}\right)_{k \geq-1}$ satisfies the third equation in (54) and

$$
\hat{y}_{-1}=\hat{y}_{0}=0, \quad \hat{y}_{1}=1 \text {. }
$$

This and $\hat{a}_{0}=1$ imply

$$
\hat{y}_{k}=\sum_{j=0}^{k-1} \hat{a}_{j} .
$$

Since (33) also holds, using the condition $a c=b d$, we get

$$
w_{n+1}=\alpha^{d} \beta^{1-a} w_{n}^{a} w_{n-1}^{c}, \quad n \in \mathbb{N}_{0} .
$$


Similar to the above is obtained

$$
w_{n+1}=\eta^{\hat{y}_{k}} w_{n+1-k}^{\hat{a}_{k}} w_{n-k}^{\hat{b}_{k}}
$$

for $k, n \in \mathbb{N}, 1 \leq k \leq n+1$, where $\eta=\alpha^{d} \beta^{1-a}$, sequences $\left(\hat{a}_{k}\right)_{k \in \mathbb{N}}$, and $\left(\hat{b}_{k}\right)_{k \in \mathbb{N}}$ satisfy (49) and (54), whereas $\left(\hat{y}_{k}\right)_{k \in \mathbb{N}}$ satisfies the third equation in (54) and (60), so, formula (61) holds.

From (63) with $k=n+1$ and (34) it follows that

$$
\begin{aligned}
w_{n+1} & =\eta^{\hat{y}_{n+1}} w_{0}^{\hat{a}_{n+1}} w_{-1}^{\hat{b}_{n+1}} \\
& =\left(\alpha^{d} \beta^{1-a}\right)^{\hat{y}_{n+1}}\left(\beta w_{-2}^{c} z_{-1}^{d}\right)^{\hat{a}_{n+1}} w_{-1}^{\hat{b}_{n+1}} \\
& =\alpha^{d \hat{y}_{n+1}} \beta^{\hat{y}_{n+2}-a \hat{y}_{n+1}} z_{-1}^{d \hat{a}_{n+1}} w_{-2}^{c \hat{a}_{n+1}} w_{-1}^{c \hat{a}_{n}}, \quad n \in \mathbb{N}_{0} .
\end{aligned}
$$

The characteristic polynomial associated to the linear difference equation (58) is the following:

$$
P_{2}(\lambda)=\lambda^{2}-a \lambda-c,
$$

from which it follows that the corresponding characteristic roots are given by the formulas in (39).

Since $a_{-1}=0$ and $a_{0}=1$, then, if $a^{2} \neq-4 c$ and $a+c \neq 1$, we easily get

$$
a_{n}=\frac{\lambda_{1}^{n+1}-\lambda_{2}^{n+1}}{\lambda_{1}-\lambda_{2}}
$$

and consequently

$$
\hat{y}_{n}=\sum_{j=0}^{n-1} \frac{\lambda_{1}^{j+1}-\lambda_{2}^{j+1}}{\lambda_{1}-\lambda_{2}}=\frac{\left(\lambda_{2}-1\right) \lambda_{1}^{n+1}-\left(\lambda_{1}-1\right) \lambda_{2}^{n+1}+\lambda_{1}-\lambda_{2}}{\left(\lambda_{1}-1\right)\left(\lambda_{2}-1\right)\left(\lambda_{1}-\lambda_{2}\right)} .
$$

If $a^{2} \neq-4 c$ and $a+c=1$, then one of the characteristic roots, say $\lambda_{2}$, is equal to one, and we have

$$
a_{n}=\frac{\lambda_{1}^{n+1}-1}{\lambda_{1}-1}
$$

and

$$
\hat{y}_{n}=\sum_{j=0}^{n-1} \frac{\lambda_{1}^{j+1}-1}{\lambda_{1}-1}=\frac{n-(n+1) \lambda_{1}+\lambda_{1}^{n+1}}{\left(1-\lambda_{1}\right)^{2}} .
$$

If $a^{2}=-4 c$ and $a+c \neq 1$, then we have

$$
a_{n}=(n+1) \lambda_{1}^{n}, \quad n \in \mathbb{N}_{0},
$$


and

$$
\hat{y}_{n}=\sum_{j=0}^{n-1}(j+1) \lambda_{1}^{j}=\frac{1-(n+1) \lambda_{1}^{n}+n \lambda_{1}^{n+1}}{\left(1-\lambda_{1}\right)^{2}} .
$$

If $a^{2}=-4 c$ and $a+c=1$, then we have

$$
a_{n}=n+1, \quad n \in \mathbb{N}_{0},
$$

and

$$
\hat{y}_{n}=\sum_{j=0}^{n-1}(j+1)=\frac{n(n+1)}{2} .
$$

Using (65)-(72) into (57) and (64) and by some calculations equations (37), (38), (40), (41), (43), (44), (46), and (47) are obtained. By some standard, but time-consuming calculations, it is shown that these formulas really represent solutions to system (1) in each if these four cases.

Remark 1 Note that if $a^{2}=-4 c$ and $a+c=1$, then $(a-2)^{2}=0$, from which it follows that $a=2$ and consequently $c=-1$. Hence, equations (46) and (47) can also be written in the following, more concrete, forms:

$$
z_{n}=\alpha^{n^{2}+n+1} \beta^{b \frac{(n-1) n}{2}} z_{-1}^{2(n+1)} w_{-2}^{b(n+1)} w_{-1}^{b n}
$$

and

$$
w_{n}=\alpha^{d \frac{n(n+1)}{2}} \beta^{-\frac{(n+1)(n-2)}{2}} z_{-1}^{d(n+1)} w_{-2}^{-(n+1)} w_{-1}^{-n}
$$

\section{Competing interests}

The author declares that he has no competing interests.

Received: 5 October 2016 Accepted: 17 November 2016 Published online: 25 November 2016

\section{References}

1. Agarwal, RP: Difference Equations and Inequalities: Theory, Methods, and Applications, 2nd edn. Marcel Dekker, New York (2000)

2. Andruch-Sobilo, A, Migda, M: Further properties of the rational recursive sequence $x_{n+1}=a x_{n-1} /\left(b+c x_{n} x_{n-1}\right)$. Opusc. Math. 26(3), 387-394 (2006)

3. Berg, L, Stević, S: On some systems of difference equations. Appl. Math. Comput. 218, 1713-1718 (2011)

4. Karakostas, GL: Convergence of a difference equation via the full limiting sequences method. Differ. Equ. Dyn. Syst. 1(4), 289-294 (1993)

5. Kent, CM, Kosmala, W: On the nature of solutions of the difference equation $x_{n+1}=x_{n} x_{n-3}-1$. Int. J. Nonlinear Anal. Appl. 2(2), 24-43 (2011)

6. Papaschinopoulos, G, Fotiades, N, Schinas, CJ: On a system of difference equations including negative exponential terms. J. Differ. Equ. Appl. 20(5-6), 717-732 (2014)

7. Papaschinopoulos, G, Psarros, N, Papadopoulos, KB: On a system of $m$ difference equations having exponential terms. Electron. J. Qual. Theory Differ. Equ. 2015, Article ID 5 (2015)

8. Papaschinopoulos, G, Schinas, CJ: On a system of two nonlinear difference equations. J. Math. Anal. Appl. 219(2), 415-426 (1998)

9. Papaschinopoulos, G, Schinas, CJ: On the behavior of the solutions of a system of two nonlinear difference equations. Commun. Appl. Nonlinear Anal. 5(2), 47-59 (1998)

10. Papaschinopoulos, G, Schinas, CJ: Invariants for systems of two nonlinear difference equations. Differ. Equ. Dyn. Syst. 7(2), 181-196 (1999)

11. Papaschinopoulos, G, Schinas, CJ: Invariants and oscillation for systems of two nonlinear difference equations Nonlinear Anal. TMA 46(7), 967-978 (2001) 
12. Papaschinopoulos, G, Schinas, $\mathrm{CJ}$ : On the dynamics of two exponential type systems of difference equations. Comput. Math. Appl. 64(7), 2326-2334 (2012)

13. Papaschinopoulos, G, Schinas, CJ, Stefanidou, G: On the nonautonomous difference equation $x_{n+1}=A_{n}+\left(x_{n-1}^{p} / x_{n}^{q}\right)$. Appl. Math. Comput. 217, 5573-5580 (2011)

14. Papaschinopoulos, G, Stefanidou, G: Asymptotic behavior of the solutions of a class of rational difference equations. Int. J. Difference Equ. 5(2), 233-249 (2010)

15. Stefanidou, G, Papaschinopoulos, G, Schinas, C: On a system of max difference equations. Dyn. Contin. Discrete Impuls. Syst., Ser. A Math. Anal. 14(6), 885-903 (2007)

16. Stefanidou, G, Papaschinopoulos, G, Schinas, CJ: On a system of two exponential type difference equations. Commun. Appl. Nonlinear Anal. 17(2), 1-13 (2010)

17. Stević, S: On a generalized max-type difference equation from automatic control theory. Nonlinear Anal. TMA 72 1841-1849 (2010)

18. Stević, S: On the difference equation $x_{n}=x_{n-k} /\left(b+c x_{n-1} \cdots x_{n-k}\right)$. Appl. Math. Comput. 218, 6291-6296 (2012)

19. Stević, S: First-order product-type systems of difference equations solvable in closed form. Electron. J. Differ. Equ. 2015, Article ID 308 (2015)

20. Stević, S: Product-type system of difference equations of second-order solvable in closed form. Electron. J. Qual. Theory Differ. Equ. 2015, Article ID 56 (2015)

21. Stević, S: Solvable subclasses of a class of nonlinear second-order difference equations. Adv. Nonlinear Anal. 5(2), 147-165 (2016)

22. Stević, S, Alghamdi, MA, Alotaibi, A, Elsayed, EM: Solvable product-type system of difference equations of second order. Electron. J. Differ. Equ. 2015, Article ID 169 (2015)

23. Stević, S, Alghamdi, MA, Alotaibi, A, Shahzad, N: Boundedness character of a max-type system of difference equations of second order. Electron. J. Qual. Theory Differ. Equ. 2014, Article ID 45 (2014)

24. Stević, S, Diblik, J, Iričanin, B, Šmarda, Z: On a solvable system of rational difference equations. J. Differ. Equ. Appl. 20(5-6), 811-825 (2014)

25. Stević, S, Diblik, J, Iričanin, B, Šmarda, Z: Solvability of nonlinear difference equations of fourth order. Electron. J. Differ. Equ. 2014, Article ID 264 (2014)

26. Stević, S, Iričanin, B, Šmarda, Z: On a product-type system of difference equations of second order solvable in closed form. J. Inequal. Appl. 2015, Article ID 327 (2015)

27. Stević, S, Iričanin, B, Šmarda, Z: Solvability of a close to symmetric system of difference equations. Electron. J. Differ. Equ. 2016, Article ID 159 (2016)

28. Stević, S, Iričanin, B, Šmarda, Z: Two-dimensional product-type system of difference equations solvable in closed form. Adv. Differ. Equ. 2016, Article ID 253 (2016)

29. Stević, S, Ranković, D: On a practically solvable product-type system of difference equations of second order. Electron. J. Qual. Theory Differ. Equ. 2016, Article ID 56 (2016)

30. Jordan, C: Calculus of Finite Differences. Chelsea Publishing Company, New York (1956)

31. Krechmar, VA: A Problem Book in Algebra. Mir Publishers, Moscow (1974)

32. Levy, H, Lessman, F: Finite Difference Equations. Dover Publications, New York (1992)

33. Mitrinović, DS, Kečkić, JD: Methods for Calculating Finite Sums. Naučna Knjiga, Beograd (1984) (in Serbian)

\section{Submit your manuscript to a SpringerOpen ${ }^{\circ}$ journal and benefit from:}

- Convenient online submission

Rigorous peer review

- Immediate publication on acceptance

- Open access: articles freely available online

- High visibility within the field

- Retaining the copyright to your article 